\title{
Anemia e peso ao nascer Anemia and birthweight
}

\section{Taqueco T Uchimura ${ }^{a}$, Sophia C Szarfarc ${ }^{b}$, Maria R D de O Latorre $^{c}$, Nelson S Uchimura ${ }^{d}$ e Sonia Buongermino de Souza}

a Departamento de Enfermagem da Universidade Estadual de Maringá. Maringá, PR, Brasil.

${ }^{b}$ Departamento de Nutrição da Faculdade de Saúde Pública da Universidade de São Paulo. São Paulo, SP, Brasil. 'Departamento de Epidemiologia da Faculdade de Saúde Pública da Universidade de São Paulo. São Paulo, SP, Brasil. 'Departamento de Medicina da Universidade Estadual de Maringá. Maringá, $P R$, Brasil

\section{Descritores}

Recém-nascido de baixo peso. Anemia. Desnutrição protéico-energética. Crescimento. Peso-estatura.

\section{Keywords}

Anemia. Infant, low birth weight. Protein-energy malnutrition. Growth. Height weight.

\section{Resumo}

\section{Objetivo}

Verificar a influência do baixo peso ao nascer (BPN) na anemia e desnutrição da criança, ao longo do primeiro ano de vida.

\section{Métodos}

A população amostral foi constituída por todas as crianças menores de um ano de idade atendidas nas unidades de saúde do Município de Maringá, PR, em 1998, num total de 587. Considerou-se baixo peso ao nascer, todas as crianças com peso $<2.500$ g. $\mathrm{O}$ estudo do crescimento para o indicador peso/idade e altura/idade teve como referência o padrão NCHS (National Center for Health Statistics). Para o diagnóstico da anemia, utilizou-se a dosagem bioquímica da concentração de hemoglobina pelo método colorimétrico direto, HemoCue. Foi considerada anêmica toda a criança com $[\mathrm{Hb}]<11,0 \mathrm{~g} / \mathrm{dL}$.

\section{Resultados}

Da população total, $58 \%$ eram crianças anêmicas e 37 (6,3\%) apresentaram baixo peso ao nascer; a anemia foi mais prevalente no segundo semestre de vida $(p=0,0093)$. A desnutrição identificada pelo índice altura/idade apresentou-se elevada especialmente para as crianças de zero a três meses nascidas de baixo peso.

Conclusões

O índice de baixo peso ao nascer na população estudada apresenta um percentual semelhante ao de países desenvolvidos. Sugere-se a implementação de um serviço de pré-natal para as mulheres de risco visando à redução desse evento que afeta a criança, dificultando o seu crescimento e aumentando o risco de anemia e suas inúmeras conseqüências deletérias.

\section{Abstract}

\section{Objective}

To verify the influence of Low Birthweight $(L B W)$ on child anemia and malnutrition during the first year of life.

\section{Methods}

Sample population included all children under one year seen at Health Units of the municipality of Maringá, southern Brazil, in 1998. Total sample size was 587 children. $L B W$ was defined as birthweight below $2500 \mathrm{~g}$. The analysis of growth for the weighfor-age and height-for-age indicators was based on NationalCenter for HealthSstatiscs

*Parte da tese de doutorado apresentada a Faculdade de Saúde Pública da USP, em 2000. Recebido em 16/7/2001. Reapresentado em 10/3/2003. Aprovado em 7/4/2003. 
standards. For anemia diagnosis, a biochemical hemoglobin concentration dosage, using Hemo Cue direct colorimetric method was employed. Children with [Hb] $<11,0$ $\mathrm{g} / \mathrm{dL}$ were considered as anemic.

\section{Results}

Fifty-eight percent of the studied population were anemic, and 37 children (6.3\%) presented LBW. Anemia was more prevalent during the second semester of life ( $p=0.0093)$. Undernutrition, as indicated by the height-for-age indicator, was high especially for children aged 0-3 months with $L B W$.

\section{Conclusions}

Although LBW rates among the studied population were similar to those of developed countries, we suggest the implementation of specific antenatal care for high-risk women, aiming at reducing $L B W$, an event that affects the child, hampering its growth and increasing the risk of anemia and its countless deleterious consequences.

\section{INTRODUÇÃO}

A Organização Mundial da Saúde (OMS, $\left.{ }^{10} 1993\right)$ classifica baixo peso ao nascer (BPN), o recém-nascido com peso menor que $2.500 \mathrm{~g}$, constituindo um dos mais importantes fatores de predição da sobrevivência infantil (Puffer \& Serrano, $\left.{ }^{12} 1988\right)$. As conseqüências deletérias decorrentes do BPN para a sobrevida e desenvolvimento da criança, têm sido sobejamente descritas. Compete-se ao pré-natal minimizar os riscos de incidência desse evento; e à puericultura dar um atendimento especial a essa criança de forma a recuperá-la, colocando-a em condições de igualdade com crianças nascidas com peso adequado e de termo.

O crescimento é um processo que sofre alterações rápidas durante a infância e é afetado por vários fatores como o sexo, o comprimento ao nascer e o potencial genético. A avaliação do crescimento define a saúde e o estado nutricional, porque os distúrbios de saúde, sem considerar a sua etiologia, afetam, invariavelmente, o crescimento infantil (Onis \& Habitch, $\left.{ }^{11} 1996\right)$.

A anemia, ao lado da desnutrição, afeta o estado nutricional da criança. $\mathrm{O}$ termo anemia se refere à anemia ferropriva. A deficiência de ferro traz conseqüências deletérias para a saúde dos indivíduos, diminuindo a capacidade física, influindo negativamente na capacidade laborativa, tanto individual quanto para a comunidade.

Durante a gestação a deficiência de ferro resulta na redução do estoque de ferro, acarretando a anemia na criança (Ionemoto \& Petlik, ${ }^{4}$ 1992). Segundo Lonnerdal \& Dewey ${ }^{6}$ (1996), até os quatro meses de vida, existe uma alta concentração de hemoglobina circulante e o ferro é obtido pela destruição dos eritrócitos e reutilizado para a síntese de novas hemoglobinas, reduzindo, dessa forma, a necessida- de de ferro exógeno. Acrescente-se também que a criança apresenta grande reserva de ferro no fígado até esta fase. Segundo estimativas, $25 \%$ a $30 \%$ da população mundial têm carência de ferro, com altas prevalências observadas entre lactentes e pré-escolares, chegando a alcançar níveis de $70 \%$ nos menores de 12 meses de idade. Brunken ${ }^{1}$ (1999), em estudo realizado no Município de São Paulo, relata aumento dessa prevalência, principalmente em crianças menores de um ano. A partir dos 12 aos 24 meses, observa-se uma diminuição da prevalência da anemia, devido à diminuição na velocidade de crescimento (Olivares et al, ${ }^{8}$ 1999).

A concentração do nível de hemoglobina para o diagnóstico de anemia ferropriva, mesmo na ausência de outros exames bioquímicos, é considerada adequada em populações onde a deficiência de ferro apresenta alta prevalência. Diante do exposto e dos diferentes fatores que interferem no estado nutricional da criança e, principalmente, seu significado para a evolução do infante, o presente estudo teve por objetivo identificar a prevalência da anemia e o estado nutricional das crianças no primeiro ano de vida, levando em consideração o peso ao nascer.

\section{MÉTODOS}

Realizou-se estudo transversal na cidade de Maringá, PR, em Postos de Saúde da Secretaria de Saúde, que se distribuem por todo o município para facilitar o acesso da população aos serviços de saúde. As atividades desenvolvidas são as de consultas médicas, enfermagem e consultas odontológicas, direcionadas a crianças, gestantes e adultos.

Para a população de estudo foram incluídas as crianças menores de um ano de idade, não gemelares e atendidas nos Postos de Saúde durante um período de cinco dias úteis. Essa inclusão foi realizada no período de março a julho de 1998, totalizando 587 crianças. 
As entrevistas com as mães, que acompanhavam as crianças, foram realizadas por pessoas devidamente treinadas; em casos onde a criança comparecia sem a mãe, sua presença era solicitada por meio de carta convite.

O crescimento da população amostral foi avaliado de acordo com padrão do "National Center for Health Statistics" (NCHS), ${ }^{7}$ recomendado pela $\mathrm{WHO}^{18}(1995)$, e os índices utilizados foram a altura para a idade (A/I) e o peso para a idade (P/I). O estado nutricional das crianças foi classificado utilizando-se os sistemas de classificação em escore Z. Esse escore representa o número de desvios-padrão, acima ou abaixo da mediana da população de referência, que corresponde ao peso ou à altura da criança. Definiu-se menos dois escores $Z$ como nível de corte mais adequado para classificar a desnutrição energético-proteíca (WHO, $\left.{ }^{18} 1995\right)$.

As crianças foram divididas segundo o peso ao nascer: BPN (baixo peso ao nascer $<2.500 \mathrm{~g}$ ); PIN (peso insuficiente $\geq 2.500 \mathrm{~g}$ a $2.999 \mathrm{~g}$ ); PAD (peso adequado $\geq 3.000 \mathrm{~g}$ ); RCIU (retardo de crescimento intra-uterino). Do total da amostra, 7 (1,19\%) mães não souberam informar o comprimento ao nascer de suas crianças.

Para o diagnóstico da anemia utilizou-se a dosagem bioquímica da concentração de hemoglobina [Hb], com valores críticos de $11,0 \mathrm{~g} / \mathrm{dL}$ para as crianças e $11,9 \mathrm{~g} / \mathrm{dL}$ para as mães (OMS, $\left.{ }^{9} 1975\right)$. A [Hb] foi medida no HemoCue - hemoglobinômetro portátil.

Comparou-se a $[\mathrm{Hb}]$ observada com a [Hb] estimada, para verificar a influência do crescimento sobre a concentração da hemoglobina. Essa influência pôde ser analisada através de um cálculo teórico da concentração da hemoglobina, considerando-se a quan- tidade de ferro veiculada pelo alimento, em relação a quantidade endógena existente no nascimento.

Foi criado um banco de dados no Dbase III de todas as variáveis codificadas e, posteriormente, foram analisadas nos softwares Epi-Info. Para a análise entre as variáveis de estudo e a presença de baixo peso ao nascer, foram realizados os testes de associação pelo qui-quadrado com correção de Yates, análise ajustada de Mantel - Haenzel e a medida de associação linear entre as variáveis quantitativas, pelo teste de correlação de Pearson. Em todas as análises utilizouse o nível de significância $\alpha=5 \%$.

\section{RESULTADOS}

Do total de 587 crianças estudadas menores de um ano de idade, $315(53,6 \%)$ eram do gênero masculino. A anemia apresentou-se com uma prevalência de 58\% das crianças e, em relação à idade, ela é mais prevalente no segundo semestre de vida, sendo esta diferença estatisticamente significativa $(\mathrm{p}=0,0093)$ (Tabela 1$)$. Do total $4(0,68 \%)$ amostras de exames de sangue apresentaram problemas técnicos e foram excluídas.

Embora o ganho de peso entre as grávidas adolescentes seja menor e a perda de peso após o nascimento seja maior, não foi observada relação da criança com a anemia materna, pois para o total das mães, $86(14,6 \%)$ foram consideradas anêmicas, não havendo, no entanto, relação desta com o BPN $(\mathrm{p}=0,6042)$ (Tabela 2).

Observou-se que as médias de concentração de hemoglobina para os grupos estudados foram de 9,7 $\mathrm{g} / \mathrm{dL}$ para as crianças BPN, 10,6 $\mathrm{g} / \mathrm{dL}$ para as de peso insuficiente, $10,6 \mathrm{~g} / \mathrm{dL}$ para aquelas de peso adequado e, para o total do estudo, o valor encontrado foi de $11,0 \mathrm{~g} / \mathrm{dL}$. E que até o $4^{\circ}$ mês de vida a [Hb] estimada

Tabela 1 - Prevalência de anemia em crianças segundo idade, Maringá, PR, 1998. (N=583)

\begin{tabular}{lrrrrrr}
\hline Idade (meses) & Anêmico & \multicolumn{2}{c}{ Não anêmico } & & Total & N \\
\hline 0-6 meses & 166 & 49,1 & 147 & 60,0 & 313 & 100,0 \\
6-12 meses & 172 & 50,9 & 98 & 40,0 & 270 & 100,0 \\
\hline Total & 338 & 58,0 & 245 & 42,0 & 583 & 100,0 \\
\hline
\end{tabular}

$\mathrm{X}^{2}=6,76 \mathrm{p}=0,0093$

Tabela 2 - Distribuição do número e percentagem de mães segundo o peso ao nascer da criança e a concentração de hemoglobina $[\mathrm{Hb}]$ da mãe. Maringá, PR, 1998. ( $\mathrm{N}=587)$

\begin{tabular}{|c|c|c|c|c|c|c|}
\hline \multirow[t]{2}{*}{ Peso ao nascer } & \multicolumn{2}{|c|}{$\begin{array}{c}{[\mathrm{Hb}]} \\
\text { Anêmica }\end{array}$} & \multicolumn{2}{|c|}{$\begin{array}{c}\text { Da mãe } \\
\text { Não anêmica }\end{array}$} & \multicolumn{2}{|c|}{ Total das crianças } \\
\hline & $\mathrm{N}$ & $\%$ & $\mathrm{~N}$ & $\%$ & $\mathrm{~N}$ & $\%$ \\
\hline $\begin{array}{l}<2.500 \mathrm{~g} \\
\geq 2.500 \mathrm{~g}\end{array}$ & $\begin{array}{r}7 \\
79\end{array}$ & $\begin{array}{l}(18,9) \\
(14,4)\end{array}$ & $\begin{array}{r}30 \\
471\end{array}$ & $\begin{array}{l}(81,1) \\
(85,6)\end{array}$ & $\begin{array}{r}37 \\
550\end{array}$ & $\begin{array}{r}(6,3) \\
(93,7)\end{array}$ \\
\hline Total & 86 & $(14,6)$ & 501 & $(85,4)$ & 587 & $(100)$ \\
\hline
\end{tabular}


é superior à observada. Em todos os três grupos estudados observou-se uma baixa correlação entre essas variáveis, contrastando com o referido na literatura.

A avaliação do estado nutricional das crianças apresentou-se diferenciada. Os grupos foram analisados segundo o peso ao nascer e os indicadores nutricionais de altura para a idade (A/I) e peso para a idade (P/I). A análise por índice antropométrico mostra a prevalência da desnutrição segundo o índice nutricional e a idade atual da criança, observando que para as crianças de zero a três meses o índice A/I se apresentou com um percentual de $14,8 \%$ (Tabela 3 ).

Para o total da população, foram encontrados $2,9 \%$ de desnutridos para o índice peso/idade, 3,3\% para peso/altura e 10,9\% para o índice altura/idade. As crianças BPN apresentaram os percentuais mais elevados para a altura/idade e para o peso/idade (Tabela 4).

Analisando o grupo de crianças BPN, observouse que, tanto para as crianças com retardo de crescimento intra-uterino, quanto para as crianças prematuras, o índice de desnutrição A/I é alto, sendo 52,9\% e $30,0 \%$, respectivamente. Para as prematuras encontramos $25,0 \%$ de desnutrição P/I e 5,0\% de desnutrição pelo índice peso/altura, resultados similares ao estudo de Victora et $\mathrm{al}^{17}$ (1989), diferenciado, no entanto, para os casos de desnutrição para o peso/ idade (Tabela 5).

\section{DISCUSSÃO}

Em estudos populacionais, o BPN e a anemia estão sempre relacionados, pois o peso ao nascer é um fator muito importante na determinação da anemia e, quando ocorre um crescimento mais acelerado, a anemia se instala. A anemia é, ao lado da desnutrição, uma deficiência de suma importância durante a gravidez, pela elevada prevalência com que ocorre e, especialmente, pelos efeitos prejudiciais a ela associados.

No presente estudo, verificou-se a importância do peso ao nascer, no estado nutricional da criança, confirmando ser essa uma condição fundamental para a sobrevivência no primeiro ano de vida.

Chama a atenção a inexistência de valores padronizados para identificação da anemia no primeiro semestre de vida. Os valores usualmente utilizados referem-se a crianças selecionadas e cuja dieta, a maior parte das vezes, incluía alimentos fortificados com ferro, prática alimentar pouco comum entre as crianças atendidas em serviços públicos de saúde. Assim, adotou-se também para o grupo de crianças de zero a seis meses inclusive, o valor de $11,0 \mathrm{~g} / \mathrm{dL}$.

Tabela 3 - Prevalência da desnutrição segundo o índice nutricional e a idade atual. Maringá, PR, 1998. (N=580)

\begin{tabular}{lccrr}
\hline Índice & & Percentual de crianças desnutridas* segundo a idade & \\
& $0-3$ meses & $4-6$ meses & $7-9$ meses & $10-12$ meses \\
\hline Altura/ldade & 14,8 & 8,9 & 10,1 & 11,2 \\
Peso/Idade & 0,9 & 2,0 & 7,0 & 3,4 \\
Peso/Altura & 2,3 & 4,1 & $128(22,1)$ & 3,4 \\
\hline Total & $217(37,4)$ & $146(25,2)$ & $89(15,3)$ \\
\hline
\end{tabular}

*Abaixo da média menos dois desvios-padrão do escore-Z (NCHS).

Tabela 4 - Prevalência da desnutrição segundo os grupos de peso ao nascer e o índice nutricional atual. Maringá, PR, 1998.

\begin{tabular}{lcccr}
\hline Peso ao nascer & & Percentual de crianças desnutridas* & Peso/Altura & Total de crianças \\
& Altura/ldade & Peso/ldade & 2,7 & 36 \\
BPN & 41,7 & 11,1 & 7,4 & 122 \\
PIN & 18,0 & 4,1 & 2,1 & 422 \\
PAD & 6,1 & 1,4 & $19(3,3 \%)$ & 580 \\
\hline Total & $63(10,9 \%)$ & $15(2,9 \%)$ & \\
\hline
\end{tabular}

*Abaixo da média menos dois desvios-padrão do escore-Z (NCHS).

$\mathrm{BPN}=$ baixo peso ao nascer

$\mathrm{PIN}=$ peso inadequado

$\mathrm{PAD}=$ peso adequado.

Tabela 5 - Prevalência da desnutrição das crianças com baixo peso ao nascer e índice nutricional atual. Maringá, PR, 1998.

\begin{tabular}{|c|c|c|c|c|c|}
\hline \multirow{2}{*}{$\begin{array}{l}\text { Peso ao } \\
\text { nascer }\end{array}$} & \multirow[b]{2}{*}{ Altura / Idade } & \multicolumn{3}{|c|}{ Percentual de crianças desnutridas* } & \multirow[b]{2}{*}{$\begin{array}{l}\text { Total de } \\
\text { crianças }\end{array}$} \\
\hline & & Peso/Idade & Peso/Altura & Normal & \\
\hline RCIU & $9(52,9)$ & $2(11,8)$ & - & $6(35,3)$ & $17(45,9)$ \\
\hline Prematura & $10(30,0)$ & $5(25,0)$ & $1(5,0)$ & $4(20,0)$ & $20(54,1)$ \\
\hline Total & $19(51,3)$ & $7(18,9)$ & $1(2,7)$ & $10(27,1)$ & $37(54,1)$ \\
\hline
\end{tabular}

RCIU $=$ retardo de crescimento intra-uterino 
No estudo da evolução da concentração da hemoglobina, tanto para a mãe, quanto para a criança, observa-se que a mãe recupera-se da gestação e puerpério muito rapidamente. Dois meses após o parto, a $[\mathrm{Hb}]$ média é similar à de mulheres não gestantes. Diferentemente do que ocorre com a mãe, as crianças diagnosticadas como anêmicas $338(58,0 \%)$ apresentaram $\mathrm{OR}=3,03(\mathrm{p}=0,0170)$ para o BPN, permitindo sugerir que a criança que nasce com peso menor que $2.500 \mathrm{~g}$ tem uma probabilidade triplicada de ser anêmica, comparada àquela com peso superior a $2.500 \mathrm{~g}$.

São inúmeras as questões referentes ao preparo da adolescente e da competição nutricional desta com o seu concepto. Essa condição pode acarretar em resultados negativos para a criança, levando ao nascimento de crianças prematuras ou com retardo de crescimento e essas não só apresentariam alterações de crescimento, como de desenvolvimento. Acrescente-se que os filhos de mães adolescentes poderiam apresentar maior risco de anemia e outros distúrbios pelo mesmo motivo (Silva et al, ${ }^{15}$ 1992).

A quantidade de ferro acumulada pelo feto na fase intrauterina é proporcional ao seu aumento de peso. Assim, quanto menor o peso de nascimento mais baixa é a quantidade de ferro orgânico. Esse fato é confirmado no presente estudo, pois dentre as crianças BPN, 29 (78,4\%) eram anêmicas e, especificamente para o grupo de RCIU, 76,5\% apresentavam deficiência.

Segundo DeMayer et $\mathrm{al}^{3}$ (1989), durante o primeiro semestre, o lactente utiliza as reservas de ferro acumuladas na vida intrauterina para suprir suas necessidades e, devido ao rápido crescimento no primeiro ano de vida, essas reservas se esgotam aos quatro meses. Esse declínio referido nos dois meses de vida foi observado no presente estudo, acrescentando-se que aos quatro meses, quando as reservas de ferro já se esgotaram, a reposição do mineral apresenta-se adequada, pois o aleitamento materno, apesar de pobre em ferro, apresenta-se com alta biodisponibilidade, sendo o alimento mais adequado para a criança nesta faixa etária.

Vários estudos foram realizados em países desenvolvidos e nenhum deles relatou um efeito significativo no estado hematológico ou ingestão de ferro, ajustado para a idade gestacional e peso ao nascer. Também não houve referência relacionada desse efeito na prematuridade e RCIU (Hingson et al, ${ }^{5}$ 1982).

Associada ao crescimento, a introdução precoce da dieta de desmame a base de legumes, cereais e leite de vaca podem levar ao desenvolvimento da anemia por volta do sexto mês, uma vez que o leite, além de ser pobre em ferro, inibe a absorção desse mineral (Dallman \& Reeves, ${ }^{2}$ 1984). Com esse objetivo, realizou-se o cálculo teórico de concentração de hemoglobina (hemoglobina estimada), com base no peso ao nascer e o peso no momento da coleta de dados, para verificar a influência do crescimento sobre a concentração da hemoglobina, como no trabalho de Sichieri ${ }^{14}$ (1987).

Considerando que durante a vida intra-uterina o feto acumula ferro em quantidade proporcional a seu aumento de peso, realizou-se a análise de correlação entre os níveis de hemoglobina observada e estimada para o grupo de crianças segundo o peso ao nascer, como uma tentativa de se verificar se essa correlação é mantida ao longo do primeiro ano de vida. Observou-se que a partir da idade de quatro meses, as concentrações de hemoglobina observadas apresentaram-se mais elevadas que as outras, possivelmente em decorrência da ingestão de ferro exógeno veiculado pelos alimentos de desmame. Mesmo assim. os valores de [Hb] são inferiores aos propostos pela $\mathrm{OMS}^{9}(1975)$.

Embora não se verifique uma correlação entre as hemoglobinas observadas e a estimadas, sabe-se que a anemia está associada ao peso da criança e o conhecimento da etiologia da anemia no primeiro ano de vida é de fundamental importância, devido às altas prevalências nos dois primeiros anos de vida da criança. A anemia da mãe pode estar associada à desnutrição, e se a insuficiente ingestão calórica é a verdadeira causa do RCIU, a falha no controle de tal ingestão poderia produzir uma associação entre a anemia e o RCIU. A anemia, entretanto, é simplesmente um marcador de nutrição pobre da mãe e não o verdadeiro determinante causal do RCIU. Assim, se a anemia não tem efeito independente no crescimento intrauterino, o uso rotineiro de suplemento férrico durante a gestação pode não ter impacto na taxa de RCIU. Sinisterra et $\mathrm{al}^{16}$ (1991), em estudo realizado em São Paulo, encontraram no final da gestação, $29,2 \%$ gestantes anêmicas e $17,2 \%$ desnutridas, não tendo sido verificada associação entre essas duas deficiências nutricionais.

A despeito da eritropoiese durante a gestação, a concentração de hemoglobina falha progressivamente até cerca da $32^{\circ}$ semana de gestação, mesmo com grandes aumentos do volume plasmático. A anemia, se grave, pode prejudicar a liberação do oxigênio para o feto e, assim, interferir no crescimento intrauterino normal e na duração da gestação. A deficiência de ferro, mesmo sem anemia, pode afetar enzimas importantes, especialmente os citocromos e, 
por meio disso, também levar a resultados adversos na gestação.

Há de se destacar que a criança BPN tende a perpetuar alguns determinantes do evento, como a baixa estatura. Entre os fatores que interferem na qualidade da gravidez e, conseqüentemente, no peso ao nascer, destacam-se as deficiências nutricionais. Verifica-se, com poucas exceções, que as crianças com baixo peso ao nascer, independente do tempo transcorrido desde o nascimento, mantém-se com pesos abaixo do peso médio para a idade (Rodrigues et $\mathrm{al},{ }^{13} 1995$ ).

Nesse contexto, os danos à saúde causados em longo prazo, nem sempre são evidentes, como no caso da anemia, fazendo-se necessário investigar o tipo de alimento utilizado para o desmame e os fatores de risco que levam a mulher a ter a criança BPN, através de estudos populacionais de diferentes abordagens metodológicas. As análises dos diferentes fatores de risco deverão levar em conta o tipo de população e a disponibilidade alimentar dessa.

Nesse sentido, um estudo com gestantes de risco poderá colaborar para a redução dos índices de BPN, evitando que a criança sofra, ao longo de sua vida, com o nascimento prematuro.

O ganho de peso médio atual entre as crianças BPN está sempre abaixo, quando comparadas às outras, ao longo do primeiro ano de vida, expondo a dificuldade de recuperação da criança que, no entanto, apresenta um perfil de evolução de crescimento, como já foi constatado, similar ao encontrado na população como um todo.

Os índices peso para idade e peso para altura não tiveram seus valores considerados, lembrando que em uma população sadia, seriam consideradas desnutridas $2,5 \%$.

Os dados obtidos, com um percentual de desnutrição de $14,8 \%$ para as crianças de zero a três meses, é bastante preocupante, pois sugere que a desnutrição pode ser resultado da desnutrição intra-uterina. A alimentação, basicamente láctea, tem um efeito limitado, o que, por sua vez, explica a prevalência da desnutrição crônica sob a aguda. No entanto, o aumento dessa prevalência, observado entre as crianças no segundo semestre de vida, de $10 \%$ para as crianças de sete a nove meses e de $11,2 \%$ para as maiores de 10 meses, alertam para a necessidade de manutenção e de cuidados especiais com a alimentação para a totalidade das crianças.

Os resultados mostram que as crianças BPN apresentam o maior percentual de desnutrição tanto para a altura/idade $(41,7 \%)$, quanto para o peso/idade $(11,1 \%)$, uma vez que o tamanho da criança é resultado do peso em que nasceu. Esse resultado mostrou que a criança BPN não conseguiu recuperar o seu peso atual para a idade.

Os percentuais menores encontrados para as crianças prematuras fazem sentido em termos biológicos, pois ela nasceu com um baixo peso por ser precoce, enquanto que as RCIU já estavam com o crescimento inadequado. No entanto, os percentuais encontrados das crianças prematuras, apesar de serem menores que o outro grupo, sugerem que o alimento recebido não estava sendo suficiente para um aumento de peso adequado. O controle da deficiência do ferro através da promoção de saúde, objetivando identificar e evitar os fatores de risco, incentiva medidas de proteção para a prevenção da anemia e de suas conseqüências. Intervenções preventivas específicas, como o uso do ferro suplementar a gestantes e crianças, fortificação dos alimentos e intervenções gerais, como educação em saúde, são ações que devem ser implementadas para o controle da deficiência do nutriente. A demora na detecção da deficiência, o custo empregado no diagnóstico de indivíduos anêmicos e do seu tratamento, trazem conseqüências que oneram o tratamento, mesmo quando comparados com outras medidas de maior custo inicial, como a fortificação de alimentos.

\section{REFERÊNCIAS}

1. Brunken GS. Avaliação da eficácia da suplementação semanal no controle da anemia em pré-escolares [tese de doutorado]. São Paulo: Curso de Pós-graduação Interunidades de Nutrição Humana Aplicada da USP; 1999.

2. Dallman PR, Reeves JD. Laboratory diagnosis of iron deficiency. In: Stekel A. Iron nutrition in infancy and childhood. New York: Nestlé/ Raven Press; 1984. p. 11-44.

3. DeMayer EM. Preventing and controlling iron deficiency anaemia trrough primary health care: a guide for heath administrators and programme managers. Geneva: WHO; 1989.

4. Ionemoto HF, Petlik MEI. Anemias carenciais. In: Marcondes E, coordenador. Pediatria básica. $8^{\circ}$ ed. São Paulo: Sarvier; 1992 p. 644-54. 
5. Hingson R, Alpert JJ, Day N, Dooling C, Kayne H, Morelock $\mathrm{S}$ et al. Effects of maternal drinking and marijuana use on fetal growth and development. Pediatrics. 1982; 70:539-546.

6. Lönnerdal B, Dewey GK. Epidemiologia da deficiência de ferro no lactente e na criança. Anais Nestlé 1996;52:11-7.

7. [NCHS] National Center for Health Statistics. Growth curves for children, birth - 18 years, United States. Vital Health Sta 1977;11:1-74.

8. Olivares M, Walter T, Herttrampf E, Pizarro F. Anemia and iron deficiency diseadi in children. Br Med Bull 1999;55:534-43.

9. [OMS] Organizacion Mundial de la Salud. Lucha contra la anemia nutricional especialmente contra la carencia de hierro. Ginebra; 1975. (OMS - Serie Informes Técnicos, 580).

10. [OMS\} Organização Mundial da Saúde. Classificação Internacional das Doenças. $10^{\circ}$ revisão II-5: definições, regulamentações, regras, normas para mortalidade e morbidade. São Paulo: Centro Brasileiro de Classificação de Doenças, 1993. [Dados inéditos].

11. Onis M, Habicht JP. Antropometric reference data for international use: recommentations from a World Health Organization Expert Committee. Am J Clin Nutr 1996;64:650-8.
12. Puffer RR, Serrano CN. Características del peso ao nascer. Washington (DC): Organizacion

Panamericana de la Salud; 1988. p. 89-95. (OPAS Publicacion Cientifica, 504).

13. Rodrigues C, Regidor E, Gutierrez Fisac JL. Low birth weigth in Spain associated with sociodemographic factors. J Epidemiol Community Health 1995;49:38-42.

14. Sichieri R. Anemia nutricional em crianças de 5 anos do Município de São Paulo: papel da dieta na determinação de sua prevalência [tese de doutorado]. São Paulo: Faculdade de Saúde Pública da USP; 1987.

15. Silva AAM, Gomes UA, Bettiol H, Dalbo CMR, Mucilo G, Barbieri MA. Associação entre idade, classe sopcial e hábito de fumar com o peso ao nascer. Rev Saúde Pública 1992;26:150-4.

16. Sinisterra ROT, Szarfarc SC, Benicio MHD'A. Anemia e desnutrição maternas e sua relação com peso ao nascer. Rev Saúde Pública 1991;25:193-7.

17. Victora CG, Barros FC, Vaughan JP, Lombardi C, Béria $\mathrm{JU}$, Victora MFB et al. Epidemiologia da desigualdade. $2^{\circ}$ ed. São Paulo: Hucitec; 1989.

18. [WHO] World Health Organization. Physical status: the use and interpretation of anthropometry: report of a Expert Commitee. Geneva; 1995. (Technical Report Series, 854). 